\title{
Study of megakaryocytes in bone marrow aspiration smears in patients with thrombocytopenia
}

\author{
Choudhary $\mathrm{PK}^{1}$, Sing $\mathrm{SK}^{1}$, Basnet R $\mathrm{B}^{1}$ \\ ${ }^{I}$ Department of Pathology, National Academy of Medical Sciences, Bir Hospital, Kathmandu, Nepal
}

\author{
Keywords: \\ Megakaryocytes; \\ Thrombocytopenia; \\ Myelodysplastic syndrome
}

\begin{abstract}
Background: Dysplastic changes in megakaryocyte are well-recognized features of myelodysplastic syndrome. However, several studies have shown it's occurrence in various other disorders. This study was done to understand the various megakaryocytic alterations including the dysplastic forms in hematological disorders presenting with thrombocytopenia other than in myelodysplastic syndrome.
\end{abstract}

Materials and Methods: All cases of thrombocytopenia in the study period of May 2010 to April 2012 were included. Bone marrow aspiration study was done in each case of thrombocytopenia. Megakaryocytes were examined in bone marrow aspiration smears in terms of their number and morphology. Dysplasia was considered significant if $\geq 10 \%$ of megakaryocyte examined show dysplastic morphology.

Results: Dysplastic megakaryocytes were observed in $52.3 \%, 50 \%$ and $21.2 \%$ of the cases of megaloblastic anemia, acute leukemia and immune thrombocytopenic purpura respectively. Most common dysplastic feature observed were multiple separate nuclei $(25.2 \%)$ and micromegakaryocyte (17.3\%). Hypogranular form was the least observed dysplastic feature $(1.4 \% ; 2 / 139$ cases $)$.

Conclusion: Dysplasia in megakaryocyte is a quiet common occurrence in various non-myelodysplastic syndroms related thrombocytopenia. The mere presence of dysplastic megakaryocyte should not prompt an interpretation of myelodysplastic syndroms and should always be correlated with patient's clinical and other hematological parameters.

\section{INTRODUCTION}

Thrombocytopenia is a very common hematological presentation for which a bone marrow aspiration is often sought. It has been defined as platelet count less than 150,000 / $\mathrm{mm}^{3} .^{1}$ Patients usually present with features of superficial bleeding including petechiae, echymosis, epistaxis and gum/vaginal bleeding. Thrombocytopenia can be isolated

\section{Correspondence:}

Dr. Prabesh Kr. Choudhary, MD

Department of Pathology, Nobel Medical College, Biratnagar, Nepal

Email:prabeshc@yahoo.com or be associated with bicytopenia or pancytopenia. ${ }^{1}$ Immune thrombocytopenic purpura (ITP) is a very common cause of thrombocytoepenia. Various other haematological conditions such as megaloblastic anemia, aplastic anemia and leukemias can also present with thrombocytopenia.

Since a number of different artifacts can produce pseudo-thrombocytopenia, peripheral blood smear examination is often the initial step in evaluation to look for features of pseudo-thrombocytopenia such as platelet clumping, satellitism and phagocytosis by neutrophils. ${ }^{2}$ Normal maturation and platelet formation results from 
megakaryocytic deoxyribonucleic acid (DNA) replication that occurs without cell division resulting in large lobulated, polypoid nucleus. ${ }^{1}$ A wide variety of growth factors like thrombopoietin act synergistically with other hematopoietic cytokines and transcriptional factors including HOX factors, AML-1 and NJ-1 which stimulate the maturation and growth of megakaryocytes. ${ }^{1}$ Defect in any of these stages of megakaryocytopoiesis can lead to dysmegakaryocytopoiesis and thrombocytopenia.

Dysmegakaryocytopoiesis is characterized by various megakaryocytic alterations in bone marrow aspirates and include both dysplastic and non-dysplastic features. Dysplastic features of megakaryocytes are multiple separated nuclei, micromegakaryocytes (size of large lymphocyte/monocyte with single or bilobed nucleus) and hypogranular forms (megakaryocyte with pale grey or water clear cytoplasm and sparse or no granules). ${ }^{3}$ Nondysplastic features include immature forms, emperipolesis, budding, cytoplasmic vacuolization and bare nuclei. Immature megakaryocytes are young forms with scant bluish cytoplasm and lack lobulation. Megakaryocytes are considered to show budding if there is blebbing of cytoplasm on their surface. Emperipolesis has been defined by the presence of other cell within the cytoplasm of megakaryocyte. $^{3}$

Dysplastic megakaryocytic alterations are well known in thrombocytopenia associated with myelodysplastic syndrome (MDS). However, several studies have described it's occurrence in non-myelodysplastic haematological conditions but scant data exist on their prevalence.

Thrombocytopenia, either isolated or in association with bicytopenia or pancytopenia is one of the commonly encountered haematological problems for which a bone marrow study is usually performed. Bone marrow aspiration in thrombocytopenia is particularly helpful in differentiating the hypoproliferative causes e.g. aplastic anemia from that due to peripheral destruction of the platelet.

Initially the study was designed to compare various megakaryocytic alterations between MDS and non-MDS related thrombocytopenia which could not be performed due to the mere absence of MDS in the study period. This study was thus restricted to study the prevalence of various megakaryocytic alterations in non-MDS related thrombocytopenia.

\section{MATERIALS AND METHODS}

The study was a hospital based prospective observational study done in National Academy Medical Science, Bir Hospital from May 2010 to April 2012. All patients presenting with platelet count less than $150,000 / \mathrm{mm}^{3}$ were included in the study. A written consent was taken for every case. All the relevant clinical data were collected. Manual platelet count was performed and and Giemsa stained fresh capillary blood films were prepared for every case to look for evidence of pseudo-thrombocytpenia such as platelet agglutination, satelletosis and phagocytosis by other cell. ${ }^{2}$ Cases showing evidence of psedo-thrombocytopenia or receiving chemo- or radiotherapy were excluded from the study. Under all aseptic measures, bone marrow aspiration was then performed for every case as per standard methodology. ${ }^{4}$ The bone marrow aspiration smears were then stained with Giemsa stain. ${ }^{4}$

The bone marrow smears were examined as per the standard guideline and the findings were documented. Number of megakaryocyte was expressed as the number per 10 low power fields (LPFs) and were further subdivided in to absent (0/10 LPFs), decreased (1/5-10LPFs), normal (1/1-3 $\mathrm{LPFs})$ and increased $(>2 / \mathrm{LPF}) .{ }^{3}$ At least 30 megakaryocytes were evaluated for megakaryocytic alterations including both dysplastic features (multiple separate nuclei, micromegakaryocyte and hypogranular forms) and nondysplastic features (emperipolesis, immature, bare nuclei, cytoplasmic vacuolization and budding). Dysplastic alterations were considered significant only when $10 \%$ or more of the megakaryocyte observed show the change. ${ }^{5}$

\section{RESULTS}

Out of 139 cases of thrombocytopenia, 86 cases $(61.9 \%)$ were observed in male and 53 cases $(38.1 \%)$ were in female. Thrombocytopenia was most common in the age group of $20-39$ years $(38.8 \%$; 54 cases) followed by that in $60-79$ years of age group ( $24.5 \%$; 34 cases).

Table 1 shows common causes of thrombocytopenia. The commonest cause was megaloblastic anemia (31.7\%) followed by acute leukemia $(27.3 \%)$ and ITP $(23.7 \%)$.

Average number of megakaryocyte in megaloblastic anemia was 18.34 and was normal in $47.4 \%$ of the cases. Out of 33 cases of ITP, 31 (91\%) had increased number of megakaryocytes, the average being 53.45. Table 2 shows average number of megakaryocyte per 10 LPFs in bone

Table 1: Common causes of thrombocytopenia

\begin{tabular}{lcc}
\hline Bone marrow aspiration & Number of cases & Percent \\
\hline Megaloblastic anemia & 44 & $\mathbf{3 1 . 7}$ \\
ITP & 33 & 23.7 \\
Acute leukemia & 38 & 27.3 \\
Aplastic anemia & 13 & 9.4 \\
Kalaazar & 4 & 2.9 \\
Idiopathic hypereosinophilia & 3 & 2.2 \\
Multiple myeloma & 2 & 1.4 \\
Iron deficiency anemia & 2 & 1.4 \\
\hline Total & $\mathbf{1 3 9}$ & $\mathbf{1 0 0}$ \\
\hline
\end{tabular}


Table 2: Average number of megakaryocytes per 10 LPFs

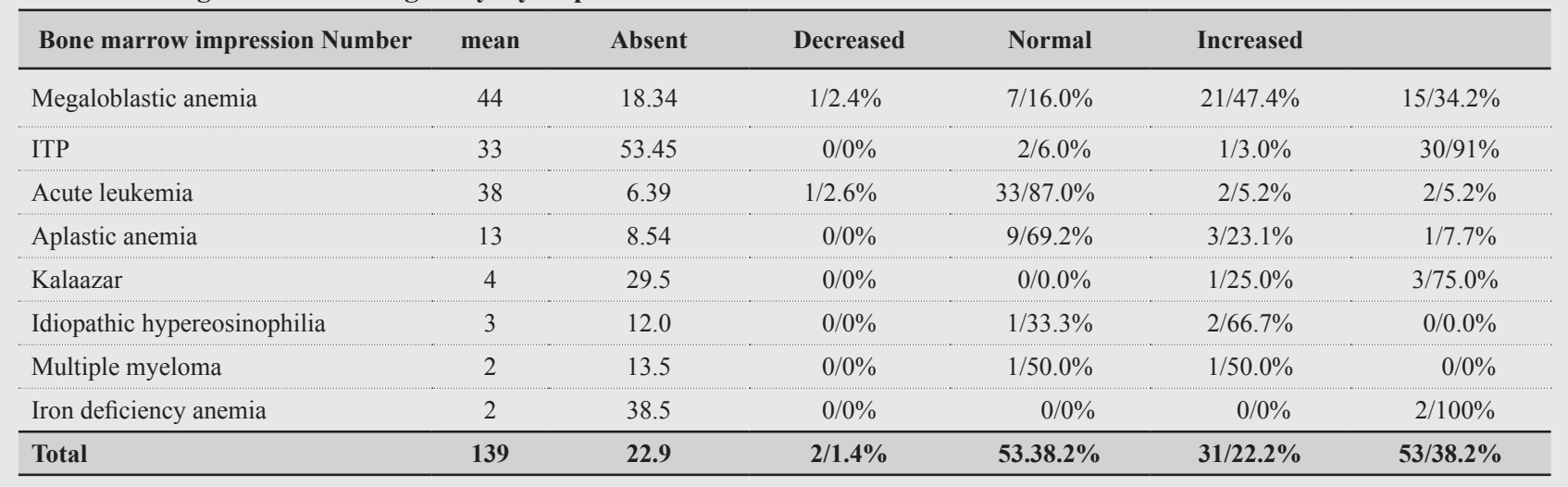

Table 3: Prevalence of dysplastic and non-dysplastic changes in various hematological disorders

\begin{tabular}{lccc}
\hline Bone marrow impression & Dysplasia & $\begin{array}{c}\text { Non- } \\
\text { Dyspiasia }\end{array}$ & Total \\
\hline Megaloblastic anemia & $23 / 52.3 \%$ & $21 / 47.7 \%$ & 44 \\
ITP & $7 / 21.2 \%$ & $26 / 78.8 \%$ & 33 \\
Acute leukemia & $19 / 50 \%$ & $19 / 50 \%$ & 38 \\
Aplastic anemia & $4 / 30.8 \%$ & $9 / 69.2 \%$ & 31 \\
Kalaazar & $4 / 100 \%$ & $0 / 0 \%$ & 4 \\
Idiopathic hypereosinophilia & $0 / 0 \%$ & $3 / 100 \%$ & 3 \\
Multiple myeloma & $0 / 0 \%$ & $2 / 100 \%$ & 2 \\
Iron deficiency anemia & $0 / 0 \%$ & $2 / 100 \%$ & 2 \\
\hline Total & $\mathbf{5 7 / 4 1 \%}$ & $\mathbf{8 2 / 5 9 \%}$ & $\mathbf{1 3 9}$ \\
\hline
\end{tabular}

marrow aspiration. Dysplastic megakaryocytes in various hematological disorders are shown in table 3. Megaloblastic anemia was more likely to have dysplastic megakaryocyte $(52.3 \% ; n=23)$ followed by acute leukemia $(50 \% ; n=19)$ and aplastic anemia $(30.8 \% ; n=4)$.

Multiple separate nuclei $(25.2 \% ; n=35)$ were the most prevalent dysplastic megakaryocytes followed by micromegakaryocyte $(17.3 \% ; n=24)$. Hypogranular forms were seen in only 2 cases $(1.4 \%)$.

Micromegakaryocyte was most prevalent in acute leukemia $(34.2 \% ; n=13)$ followed by that in megaloblastic anemia ( 5 /44 cases) and aplastic anemia (3 out of 13 cases). Multiple separate nuclei was most commonly found in megaloblastic anemia $(41 \% ; n=18)$ followed by ITP $(18 \% ; n=5)$ and acute leukemia $(16 \% ; n=6)$. Hypogranular megakaryocytes were found only in 2 cases (one in megaloblastic anemia and another one in acute leukemia).

Similarly, immature forms of megakaryocyte were also observed. Almost $94 \%$ of cases of ITP showed immature megakaryocyte. Next common cause was aplastic anemia (77\%) followed by megaloblastic anemia (50\%). Whereas, $63 \%$ cases of acute leukemia and $50 \%$ cases of kalaazar showed immature megakaryocytes. In case of idiopathic hypereosinophilia, multiple myeloma and iron deficiency
Table 4: Prevalence of emperipolesis in various hematological disorders

\begin{tabular}{lccc}
\hline Bone marrow impression & Absent & Present & Total \\
\hline Megaloblastic anemia & 43 & 1 & 44 \\
ITP & 27 & 6 & 33 \\
Acute leukemia & 36 & 2 & 38 \\
Aplastic anemia & 8 & 5 & 31 \\
Kalaazar & 3 & 1 & 4 \\
Idiopathic hypereosinophilia & 1 & 2 & 3 \\
Multiple myeloma & 2 & 0 & 2 \\
Iron deficiency anemia & 1 & 1 & 2 \\
\hline Total & $\mathbf{1 2 1}$ & $\mathbf{1 8}$ & $\mathbf{1 3 9}$ \\
\hline
\end{tabular}

anemia didn't reveal immature megakaryocytes.

Table 4 shows prevalence of emperipoleisis. Out of 13 cases of aplastic anemia emperipolesis was found in 5 cases. Emperipolesis was also found in 6 out of 27 cases of ITP.

Cytoplasmic vacuolization was observed in 5 cases of ITP only and was found in none of the other cases. Similarly, platelet budding was found in only one case of ITP and none in others.

\section{DISCUSSION}

A total of 139 cases of true thrombocytopenia were included in this study. The most common cause of thrombocytopenia was megaloblastic anemia $(31.7 \% ; n=44)$ followed by acute leukemia $(27.3 \% ; n=38)$ and $\operatorname{ITP}(23.7 \% ; n=33)$. In contrast the most common cause of thrombocytopenia in a study performed by Muhury M et al was AML(18.8\%) followed by ITP $(13.15 \%)$ and ALL (12.5\%). ${ }^{3}$ Thrombocytopenia was the most common occurrence in 20-39 years of age group $(38.8 \% ; n=54)$. In contrast Muhury $M$ et al found it most common in children less than 10 years of age followed by that in 21-30 years of age group. ${ }^{3}$ This may be due to the large number of pediatric cases going to the children hospital in out set up. Thrombocytopenia was more common in males $(61.9 \%)$ than in females $(38.1 \%)$ similar to that 
observed by Muhury $\mathrm{M}$ et $\mathrm{al}^{3}$

Out of 33 cases of ITP, 31 (91\%) had increased number of megakaryocyte in bone marrow aspiration smears, the average being 53.45 per 10 LPFs. Shi XD et al also found increased megakaryocyte in $98 \%$ of the cases of ITP. ${ }^{6}$ Whereas Muhury $\mathrm{M}$ et al found it in $94.7 \%$ of the cases. ${ }^{3}$ Jubelirer SJ et al also found increased megakaryocyte count in $95.3 \%$ of the cases of ITP. ${ }^{7}$ According to them this can be due to stimulation of the marrow megakaryocytes to synthesize platelets at an increased rate due to immunemediated platelet destruction in the spleen and other reticuloendothelial tissues. The average megakaryocyte count per 10 LPFs in acute leukemia and aplastic anemia were decreased in $87 \%$ and $69 \%$ of the cases respectively which was also found by other authors. ${ }^{3,8,9}$ Decreased megakaryocyte, according to them can be due to significant bone marrow suppression, inhibition of DNA synthesis and ineffective proliferation in case of aplastic anemia and acute leukemia.

Out of 44 cases of megaloblastic anemia, dysplastic megakaryocytes were found in 23 cases (52.3\%) which is in contrast to that found by Muhury $M$ et al $(75 \%){ }^{3}$ In a similar study done by Shalatha NP et al dysplastic megakaryocyte was found in $100 \%$ of the cases of megaloblastic anemia and suggested further investigations as they can be mistaken for MDS. ${ }^{10}$ The most common dysplastic changes in megaloblastic anemia were multiple separate nuclei $(40.9 \% ; n=18)$ and micromegakaryocyte $(11.4 \% ; n=5)$ which were also found by Muhury $M$ et al. ${ }^{3}$ Wikramasinghe et al. also found multiple separate nuclei as the most common dysplastic feature and attribute this to diminished and ineffective DNA synthesis leading to nuclear maturation defect. ${ }^{11}$ Hypogranular form was evident in 1 case $(2.3 \%)$ only. The mechanism for such dysmegakaryocytopoesis characterized by abnormal morphology according to C Wang JYR et al, is based on the impaired DNA synthesis and methylation due to folate and vitamin B12 deficiency. ${ }^{12}$ The finding of emperipolesis $(2.3 \%)$ in megaloblastic anemia was in agreement with the observation done by Tavassoli et al. ${ }^{13}$ Immature forms and bare nuclei were found in 50\% (22 cases) and 56.8\% (25 cases) respectively, similar findings were also found by Muhury $\mathrm{M}$ et al ( $42 \%$ and $58 \%$ respectively). ${ }^{3}$

Dysplastic megakaryocytes were found in 7 cases $(21.2 \%)$ only out of the 33 cases of ITP which is in contrast to that found by Muhury M et al $(89.5 \%) .{ }^{3}$ Multiple separate nuclei and micromegakaryocyte were found in 5 cases $(15.2 \%)$ and 2 cases $(6.1 \%)$ respectively. In contrast micromegakaryocyte was found in $98 \%$ of the cases of ITP by Shi XD et al. ${ }^{6}$ Sashida $G$ et al had found 2 cases mistakenly diagnosed as ITP in 13 cases of MDS presenting with isolated thrombocytoepenia and therefore recommended the use of cytogenetic in such difficult cases. ${ }^{14}$ In contrast Hwang Y et al found clonal cytogenetic abnormalities in 3 out 100 cases of morphologically normal ITP and therefore recommended routine use of cytogenetic in suspected cases of ITP. ${ }^{15}$

A marked shift to young, immature, hypolobulated megakaryocytes was the outstanding morphological feature noted in 94\% (31 cases) of ITP cases. Similar findings were also observed by Muhury $\mathrm{M}$ et al. ${ }^{3}$ Houwerzijl et al attributed this to the apoptotic and para-apoptotic type of programmed cell death (PCD) of mature megakaryocytes. ${ }^{16}$ According to them inappropriate PCD of mature megakaryocytes can disrupt platelet formation causing apoptosis-like PCD (para-apoptosis) leading to thrombocytopenia in ITP. The immature forms of megakaryocyte often resemble micromegakaryocyte which can mislead to an interpretation of myelodysplastic syndrome presenting with isolated thrombocytopenia, thus mimicking ITP. Das $\mathrm{R}$ et al therefore suggested the use of CD 61 for the detection and quantification of megakaryocyte including the dysplastic micromegakaryocyte. ${ }^{17}$

Finding of bare nuclei in 30.3\% (10 cases) of ITP was in contrast to that found by Muhury M et al (84.2\%) and Gordon et al $(50 \%){ }^{3,18}$ Emperipolesis was seen in $18.2 \%(n=6)$ of cases of ITP which was discordant to that found by Muhury M et al (68.4\%). ${ }^{3}$ Tavossoli M et al and Sabebekhitiari HA et al postulated that in the state of heightened demand for cell delivery from marrow into the circulation, some cells take a transmegakaryocytic route to enter the circulation causing the phenomenon of emperipolesis and thus suggested megakaryocytes as a component of the marrow-blood barrier. ${ }^{13,19}$ They further attributed emperipolesis to blood loss and thus recommended to investigate the patient for unsuspected blood loss. The cytoplasmic vacuolization was seen in $15 \%(n=5)$ of ITP which according to Houwerzijl et al ultrastructurally represents mitochondrial swelling. ${ }^{16}$ Cytoplasmic vacuolization might also indicate degenerative changes such as those of apoptosis and para-apoptosis. Another plausible explanation for this according to Muhury $\mathrm{M}$ et al is autophagy to maintain cell metabolism when there is increased metabolic demand and nutrition deficiency due to increased megakaryocytopoiesis or it might be a way of sequestration and degradation of specific pathogens such as immunoglobulins. $^{3}$

Nineteen $(50 \%) 38$ cases of acute leukemia show dysplastic megakaryocyte, most common being micromegakaryocyte $(34.2 \% ; n=13)$ followed by multiple separate nuclei $(15.2 \%$; $\mathrm{n}=5$ ). Dysplastic megakaryocyte in $50 \%$ of the cases was also found by Frenkel MA et al more often in AML with $\mathrm{t}$ $(8 ; 21){ }^{8}$ Micromegakaryocyte was found in $39 \%(n=11)$ of the cases in this study. In a similar study done by Muhury et al, dysplastic megakaryocyte was found in only $26.6 \%$ $(n=12)$ of the cases, more commonly in AML $(29.6 \% ; n=8){ }^{3}$ Most common dysplastic feature in AML in their study was micromegakaryocyte $(18.5 \% ; n=5){ }^{3}$ In contrast Lee EJ et al found micromegakaryocyte in $59.4 \%$ of the cases of AML. Interestingly Lee EJ et al also found no patient 
with micromegakaryocyte associated with good prognosis chromosomal abnormalities. ${ }^{9}$ In a study done by Jinnai et al, dysplastic megakaryocyte (micromegakaryocyte and multiple separate nuclei) was found in only $10 \%$ of the cases of AML. They also found significantly lower response to chemotherapy in AML cases with dysplastic megakaryocytes. ${ }^{20}$

The most common non-dysplastic megakaryocytic change in acute leukemia was immature form $(63.2 \% ; n=24)$ followed by bare nuclei $(36 \% ; n=12)$ and emperipolesis $(6 \% ; n=2)$. The exact mechanism of these changes is not clear due to paucity of literature. However, Brandt L et al found most megakaryocytes being diploid and that polyploidy were not normally developed in acute leukemia. ${ }^{21}$ From this observation they suggested that defective differentiation of the megakaryocytes and/or their precursors analogous to what has previously been demonstrated in the myeloblasts of acute leukemia may be responsible to the dyspoetic features of megakaryocyte occurring in acute myeloid leukemia. ${ }^{21}$

Out of 13 cases of aplastic anemia in this study, dysplastic megakaryocyte was found in 4 cases $(30.8 \%)$, the most common being micromegakaryocyte $(23.1 \% ; n=3)$ followed by multiple separate nuclei $(15.4 \% ; n=2)$. In contrast Muhury $\mathrm{M}$ et al found dysplastic change in megakaryocyte only in $12.5 \%$ of the cases. ${ }^{3}$ The dysplastic forms seen were also in contrast to those of Tricot et al where megakaryocytes were of normal morphology in all cases. ${ }^{22}$ Most common non-dysplastic form of megakaryocyte in aplastic anemia was immature form $(77 \%$; 10 out of 13 cases) which was in contrast to that found by Muhury et al $(25 \%){ }^{3}$ The other non-dysplastic morphology observed were emperipolesis $(38.5 \% ; \mathrm{n}=5)$ and bare nuclei $(23.1 \% ; \mathrm{n}=3)$ which are congruent to that found by Muhury et al ( $25 \%$ each). ${ }^{3}$

Dysplastic changes were found in all 4 cases of Kalaazar $(100 \%)$; multiple separate nuclei in all cases and micromegakaryocyte in 1 case. Muhury $\mathrm{M}$ et al also found dysplastic megakaryocyte in $80 \%$ of the cases of Kalaazar. ${ }^{3}$ Abnormalities in the megakaryocyte in $83 \%$ of the cases of Kalaazar were also found in a study done by Marwaha $\mathrm{N}$ et al and attributed it due to splenic sequestration and ineffective hematopoiesis. ${ }^{23}$ The non-dysplastic changes observed in Kalaazar include bare nuclei (3/4 cases), immature form ( $2 / 4$ cases) and emperipolesis ( $1 / 4$ cases).

None of the cases of idiopathic hypereosinophilia syndrome, multiple myeloma and iron deficiency anemia in this study show dysplastic megakaryocyte. Whereas Muhury $\mathrm{M}$ et al. found dysplastic megakaryocyte in $59 \%$ of the cases of multiple myeloma. ${ }^{3}$ Emperipolesis was found in $2 / 3$ cases of idiopathic hypereosinophilia and in $1 / 2$ cases of iron deficiency anemia. Bare nuclei were found in 1 case each of idiopathic hypereosinophilia syndrome, multiple myeloma and iron deficiency anemia each.
Overall out of the 139 cases included in this study, dysplastic megakaryocytes were evident in $57 \%(\mathrm{n}=41)$ of the cases which was congruent with that found by Muhury et al (53\%). ${ }^{3}$ Most of the cases with dysplastic features were that of megaloblastic anemia (40.3\%) followed by acute leukemia $(33.3 \%)$ and ITP (12.3\%). The most common dysplastic features observed in this study overall were multiple separate nuclei $(25.2 \% ; \mathrm{n}=35)$ followed by micromegakaryocyte $(17.3 \% ; n=24)$ which were in concordance to that observed by Muhury $\mathrm{M}$ et al. ${ }^{3}$ Hypogranular form was observed in only one case of megaloblastic anemia and acute leukemia (AML) each. In the similar study done by Muhury M et al, hypogranular form of megakaryocyte was found in none of the case. ${ }^{3}$

Dysmegakaryocytopoiesis in the form of micromegakaryocyte, multiple separate nuclei and hypogranular forms have been known integral feature of myelodysplastic syndrome and have been shown in some studies to be an independent prognostic factor. Hypogranular form was the least evident (2 cases) dysplastic feature observed in this study. Wong KF et al studied to determine how frequently this feature was observed in MDS and the specificity of its occurrence. On review of archival materials, hypogranular megakaryocytes were observed in $80.3 \%$ of MDS, $30.6 \%$ of AML and $1.4 \%$ of controls. On the other hand, the other well-recognized dysmegakaryocytopoietic features (multiple separate nuclei and micromegakaryocyte), though frequent in MDS or AML, were also observed in $20 \%$ of controls. They therefore proposed including megakaryocytic hypogranulation as the most diagnostic feature of myelodysplasia in MDS. ${ }^{24}$

Dysplastic megakaryocytes were found in $52.3 \%(n=23)$ of megaloblastic anemia, 50\% (19 cases) of acute leukemia, $21.2 \%$ ( 7 cases) of ITP and $30.8 \%$ (4 cases) of aplastic anemia. Dysplastic megakaryocyte in $\geq 40 \%$ of its lineage was found in none of the case included in this study. Based upon their observation on 187 patients of MDS, Matsuda et al found significant overall survival and leukemia free survival only when dysplastic megakaryocyte in $\geq$ $40 \%$ is taken as the morphological criteria for dysplasia. Accordingly they purposed this as the new defining criteria for MDS. ${ }^{25}$

Hence, this study shows that dysplastic megakaryocyte is also found in non-MDS related thrombocytopenia and by themselves do not specify MDS; other hematological conditions causing thrombocytopenia have to be considered in the differential diagnosis. A meticulous attempt should be done to find the hypogranular form of the megakaryocyte before interpreting the case as MDS as the other features including multiple separate nuclei and micromegakaryocyte were significantly found in nonMDS related thrombocytopenia in this study. This will ally the patient's anxiety and prevent the possible inadvertent treatment. However, this study included no case of MDS for 
comparison, studies with increased sample size including cases of MDS should be done to understand the occurrence of dysplastic megakaryocyte in various non-MDS disorders.

\section{CONCLUSION}

The commonest cause of thrombocytopenia in this study was megaloblastic anemia followed by acute leukemia and ITP. Dysplastic megakaryocytes were more commonly observed in megaloblastic anemia and acute leukemia. Hypogranular forms were the least common dysplastic morphology observed in this study and should be looked for in a suspected case of MDS. Due to the very common occurrence of dysplastic megakaryocytes in non-MDS haematological disorders, the threshold for dysplasia in megakaryocyte should be raised from the recommended $10 \%$. However, further comparative study including cases of MDS has to be done to understand the significance of occurrence of the dysplastic megakaryocytes in non-MDS related thrombocytopenia.

\section{REFERENCES}

1. Marshall AL, Ernest B. William's Hematology. 7th Eds. McGrawHill Companies:New York;2006.176pp.

2. A Casonato AB, Pontara E, Dannhauser D, Lazzaro AR, Girolami A. EDTA dependent pseudothrombocytopenia caused by antibodies against the cytoadhesive receptor of platelet gpIIB-IIIA. J Clin Pathol1994;47:625-30.

3. Manas Muhury AMM, Rai S, Naik R, Pai MR, Sinha R. Megakaryocytic alterations in thrombocytopenia: A bone marrow aspiration study. Indian J Pathol Microbiol 2009;52:490-4.

4. Lewis SM, Bain BJ, Bates I.Bone marrow biopsy, In: Practical hematology.10th ed: Churchill Livingstone:Philadelphia;2006. pp116-120.

5. Swerdlow SCE, Harris N, Jaffe Eet al. WHO classification of tumors of haematopoietic and lymphoid tissue. 4th ed. Brunning RD. OA, Germing U., Beau MM., Porwit A., Bauman I. et al., editor: International Agency for Reasearch on Cancer (IARC); 2008.

6. Shi XD HT, Feng YL, Liu R, Li JH, Chen J, Wang TY. A study on micromegakaryocyte in children with idiopathic thrombocytopenic purpura. Chinese journal of pediatrics 2004;42:192-5.

7. Jubelirer SJ, Harpold R. The role of the bone marrow examination in the diagnosis of immune thrombocytopenic purpura: case series and literature review. Clin Appl Thromb Hemost 2002;8:73-6.

8. Frenkel MA, Fleishman EV, Tupitsyn NN, Volkova MA, Kulagina OE. Megakaryocytic dysplasia in acute nonlymphoblastic leukemias. Ter Arkh 1995;67:12-6.

9. Lee EJ SC, Tomiyasu T, Testa JR. Clinical and cytogenetic correlations of abnormal megakaryocytopoiesis in patients with acute leukemia and chronic myelogenous leukemia in blast crisis. Leukemia 1990;4:350-3.

10. Ashalatha N, Netravathi P, Ragupathi A, Nagarajappa A. Hemogram and bone marrow morphology in cases of pancytopenia. . The Internet Journal of Laboratory Medicine. 2010 4(2) [cited 25 June 2013 ]. Available from :http://archive.ispub.com/journal/the-internetjournal-of-laboratory-medicine/volume-4-number-2/hemogram-and- bone-marrow-morphology-in-cases-of-pancytopenia.html\#sthash. SMuztQJe.dpbs

11. Wickramasinghe SN. Morphology, biology and biochemistry of cobalamin- and folate-deficient bone marrow cells. Baillieres Clin Haematol 1995;8:441-59.

12. Wang C, JYR, Liu L. Ultrastructural study of the megakaryocytes in megaloblastic anemia. Hyperlink "http://www.ncbi.nlm.nih.gov/ pubmed/2209245" \o "Zhonghua nei ke za zhi [Chinese journal of internal medicine]." Zhonghua Nei Ke Za Zhi 1990;29;158-60.

13. Tavassoli M. Modulation of megakaryocyte emperipolesis by phlebotomy: megakaryocytes as a component of marrow-blood barrier. Blood Cells 1986;12:205-16.

14. Sashida G, Takaku TI, Shoji N et al. Clinico-hematologic features of myelodysplastic syndrome presenting as isolated thrombocytopenia: an entity with a relatively favorable prognosis. Leuk Lymphoma. 2003;44:653-8.

15. Hwang Y, Huh JW, Mun YC, Seong CM, Chung WS. Myelodysplastic syndrome mimicking idiopathic thrombocytopenic purpura.Korean J Lab Med 2010;30:105-10.

16. Houwerzijl EJ, Blom NR, van der Want JJ, Vellenga E, de Wolf JT. Megakaryocytic dysfunction in myelodysplastic syndromes and idiopathic thrombocytopenic purpura is in part due to different forms of cell death. Leukemia. 2006;20:1937-42.

17. Das R, Hayer J, Dey P, Garewal G. Comparative study of myelodysplastic syndromes and normal bone marrow biopsies with conventional staining and immunocytochemistry. Anal Quant Cytol Histol 2005;27:152-6.

18. Gordon S, Lee S. Naked megakaryocyte nuclei in bone marrows of patients with acquired immunodeficiency syndrome: a somewhat specific finding. Mod Pathol1994; 7:166-8.

19. Sahebekhitiari HA, Tavassoli M. Marrow cell uptake by megakaryocytes in routine bone marrow smears during blood loss. Scand J Haematol 1976;16:13-7.

20. Jinnai I, Tomonaga M, Kuriyama K et al. Dysmegakaryocytopoiesis in acute leukaemias: its predominance in myelomonocytic (M4) leukaemia and implication for poor response to chemotherapy. Br J Haematol 1987;66:467-72.

21. Brandt L, Levan G, Mitelman F, Sjogren U. Defective differentiation of megakaryocytes in acute myeloid leukemia. Acta Med Scand 1974;196:227-30.

22. Tricot G, Vlietinck R, Boogaerts MA et al. Prognostic factors in the myelodysplastic syndromes: importance of initial data on peripheral blood counts, bone marrow cytology, trephine biopsy and chromosomal analysis. Br J Haematol 1985;60:19-32.

23. Marwaha N, Sarode R, Gupta RK, Garewal G, Dash S. Clinicohematological characteristics in patients with kalaazar. A study from north-west India. Trop Geogr Med 1991;43:357-62.

24. Wong KF, Chan JK. Are 'dysplastic' and hypogranular megakaryocytes specific markers for myelodysplastic syndrome? $\mathrm{Br} \mathrm{J}$ Haematol1991;77:509-14.

25. Matsuda A, Germing U, Jinnai I et al. Improvement of criteria for refractory cytopenia with multilineage dysplasia according to the WHO classification based on prognostic significance of morphological features in patients with refractory anemia according to the FAB classification. Leukemia 2007;21:678-86. 